\title{
Boron neutron capture therapy as salvage treatment for recurrent papillary thyroid carcinoma-a case report
}

\author{
Yi-Ying Pan ${ }^{1}$, Shan-Fan Yao ${ }^{2}$, Ko-Han Lin ${ }^{2}$, Fong-In Chou ${ }^{3}$, Jia-Cheng Lee ${ }^{1}$, Shyh-Kuan Tai ${ }^{4}$, \\ Wen-Sheng Huang, ${ }^{2}$ Keng-Li Lan ${ }^{1}$, Yee Chao ${ }^{1}$, Yi-Wei Chen ${ }^{1}$ \\ ${ }^{1}$ Division of Radiation Oncology, Department of Oncology, ${ }^{2}$ Department of Nuclear Medicine, Taipei Veterans General Hospital, Taipei, Taiwan; \\ ${ }^{3}$ Nuclear Science and Technology Development Center, National Tsing Hua University, Hsinchu, Taiwan; ${ }^{4}$ Department of Otorhinolaryngology and \\ Head and Neck Surgery, Taipei Veterans General Hospital, Taipei, Taiwan \\ Correspondence to: Yi-Wei Chen, MD, PhD. Department of Oncology, Taipei Veterans General Hospital, No. 201, Sec. 2, Shi-Pai Rd., Taipei City, \\ Taiwan. Email: chenyw@vghtpe.gov.tw.
}

\begin{abstract}
A 69-year-old man diagnosed with left papillary thyroid carcinoma underwent partial thyroidectomy on July 18, 2017. Follow-up positron emission tomography (PET) revealed a residual tumor in the left thyroid bed, so he received salvage tomotherapy with 5,500 centigray (cGy) in 29 fractions, from September 21 to November 3, 2017. The patient also took adjuvant radioactive iodine (RAI)-131 twice. Nonetheless, follow-up imaging on June 29, 2018 revealed a residual lesion on the left side of the anterior neck. Boron neutron capture therapy (BNCT) for salvage management was performed at the Tsing-Hua Open Pool Reactor (THOR) on October 8, 2018. The patient received a total dose of $450 \mathrm{mg} / \mathrm{kg}$ of L-[410borophenylalanine] fructose via continuous intravenous infusion. The blood boron concentration was $21.27 \mathrm{ppm}$ at 2 hours post-infusion just before epithermal neutron irradiation. Mean, minimal and maximal doses of gross tumor volume (GTV) were 22.36, 19.83 and 23.60 gray equivalent (GyE), respectively. One month after BNCT, PET/magnetic resonance imaging (MRI) revealed regression of the recurrent tumor, with the maximum standardized uptake value decreasing from 17.8 to 11.7. Thyroglobulin also dropped significantly, from 55.6 to $15.1 \mathrm{ng} / \mathrm{mL}$. The patient reported no significant side effects after BNCT. So far, most investigations have been confined to applying BNCT on patients of recurrent head and neck or brain tumor. In this study, we had the opportunity to present the outstanding treatment results of BNCT in a patient with recurrent thyroid papillary carcinoma in Taiwan. Based on the case outcome, BNCT can be used as salvage treatment for recurrent or refractory thyroid cancer.
\end{abstract}

Keywords: Boron neutron capture therapy (BNCT); papillary thyroid carcinoma; salvage treatment; quality of life; positron emission tomography (PET); linear energy transfer; case report

Received: 17 January 2020; Accepted: 18 June 2020; Published: 30 September 2020.

doi: $10.21037 /$ tro-20-10

View this article at: http://dx.doi.org/10.21037/tro-20-10

\section{Introduction}

Thyroid cancer is the most common endocrine gland malignancy in Taiwan. Moreover, the prevalence of thyroid carcinoma has been increasing globally (1). According to the 2016 Taiwan Cancer Registry report, the incidence of thyroid cancer has not only increased four-fold in the past two decades, but it now accounts for $3.48 \%$ of all malignant diseases (2). Histologically, it can be divided into differentiated (papillary and follicular) and undifferentiated (anaplastic) carcinomas. Approximately $80-90 \%$ of all thyroid cancers are differentiated, with a relatively favorable prognosis compared to undifferentiated.

The treatment for differentiated thyroid carcinoma (DTC) includes primary surgery (lobectomy/total thyroidectomy) followed by remnant ablation using radioactive iodine (RAI) (3). By contrast, undifferentiated thyroid carcinoma (UTC) lacks iodide uptake and also fails 
to adequately respond to radio- or chemotherapy. Hence, considerable research, both in vivo and in vitro, has been devoted to exploring new modalities to treat UTC, such as boron neutron capture therapy (BNCT) $(4,5)$.

$\mathrm{BNCT}$ is a type of radiation therapy that selectively targets malignancy by concentrating $10 \mathrm{~B}$ compound into tumor cells. When non-radioactive isotope 10B atoms absorb thermal neutrons, they break up into a Li nucleus (7-lithium) and $\alpha$ particle (4-helium), which provides high linear energy transfer along an extremely short pathway $(<10 \mu \mathrm{m})$. This feature protects the adjacent normal tissue from destructive irradiation, enabling an immense dose gradient between the tumor cells and normal cells. In collaboration with the Nuclear Science and Technology Development Center at National Tsing Hua University, radiation oncologists at Taipei Veterans General Hospital in Taiwan have conducted multiple clinical trials since 2010 using BNCT. Most studies have tended to focus on recurrent head and neck cancer and perplexing brain tumor case.

Meanwhile, rather less attention has been paid to the application of BNCT for DTC. In some cases, recurrent DTC has lost the capability to concentrate RAI and tends to be extremely aggressive. Factors like poorly differentiated, squamous, or anaplastic foci also have a markedly detrimental effect on the prognosis of papillary thyroid carcinoma (6). Consequently, BNCT may be an alternative treatment choice for these patients. In this case study, we report the treatment experience and demonstrate the results of BNCT in a patient with recurrent thyroid papillary carcinoma in Taiwan. We present the following case in accordance with the CARE reporting checklist (available at http://dx.doi.org/10.21037/tro-20-10).

\section{Case presentation}

This 69-year-old man had recurrent papillary thyroid carcinoma. A palpable thyroid mass was first noted during annual checkup in May 2017. Fine needle aspiration revealed papillary thyroid carcinoma, conventional type. The patient then underwent bilateral total thyroidectomy and left neck dissection on July 18, 2017 at a local hospital. Surgical pathology confirmed unifocal papillary carcinoma of the left thyroid lobe, $0.6 \times 0.4 \times 0.4 \mathrm{~cm}^{3}$ in size, with minimal extra-thyroid extension. The regional lymph nodes were all negative for tumor cells $(0 / 15)$; hence, the pathological staging was $\mathrm{T} 3 \mathrm{bN} 0$, according to the American Joint Committee on Cancer TNM Staging 8th edition, 2017. There was no vascular/lymphatic/ perineural invasion. Cauterized tissue edges microscopically showed carcinoma involvement. Nonetheless, a suspicious residual mass with calcification attached to the trachea/ esophagus (clinical stage T4a) was stated by the surgeon in an outpatient clinic note. After surgery, the patient received adjuvant thyroxine sodium supplement and visited the outpatient department on a regular basis. However, a follow-up positron emission tomography (PET) scan on August 23, 2017 confirmed a residual tumor in the left thyroid bed attached to the tracheal wall, with a maximum standardized uptake value (SUVmax) of 14.6 and another nodular lesion (SUVmax: 4.8) at the left posterior neck level Va. Thus, the patient received salvage tomotherapy with 5,500 centigray (cGy) in 29 fractions to the residual tumor and lymphadenopathies, and 4,500 cGy in 25 fractions to the bilateral lower neck from September 21 to November 3, 2017 at a second local hospital. After completing radiotherapy, the patient came to our hospital for adjuvant RAI treatment. The patient received oral iodine-131 (I-131) ablation therapy twice, 30 millicuries $(\mathrm{mCi}$ ) on January 26, 2018 and $120 \mathrm{mCi}$ on June 28, 2018. I-131 whole body scan and single photon emission computed tomography (SPECT), both performed one week after each treatment course, indicated functioning remnants in the right paratracheal region. Follow-up PET/ magnetic resonance imaging (MRI) on June 29, 2018 revealed a persistent residual lesion (SUVmax: 17.8) on the left side of the anterior neck. To discuss management of the remaining tumor, a multidisciplinary team conference was held on July 19, 2018. Our otorhinolaryngologist suggested salvage laryngectomy with bilateral total thyroidectomy for the case, but the patient hesitated to accept the surgery. Therefore, BNCT was chosen in an attempt at salvage management.

First, to determine sufficient distribution of boroncontaining drug inside the tumor, the patient underwent PET using 4-borono-s2 ${ }^{18}{ }^{\mathrm{F}}$-fluoro-phenylalanine (FBPAPET) on August 10, 2018 at our Department of Nuclear Medicine. The exam revealed a tumor-to-normal ratio $(\mathrm{T} / \mathrm{N})$ of 2.22 and a tumor-to-blood ratio (T/B) of 1.67 (Figure 1). Blood was referred to the blood pool in the left ventricle. Second, in order to carry out the treatment, approval was required from the Institutional Review Board (IRB) of Taipei Veterans General Hospital (TVGH) and Taiwan's Food and Drug Administration (TFDA). After we obtained the approval, a computed tomography scan was done on October 4, 2018 for treatment planning. On October 

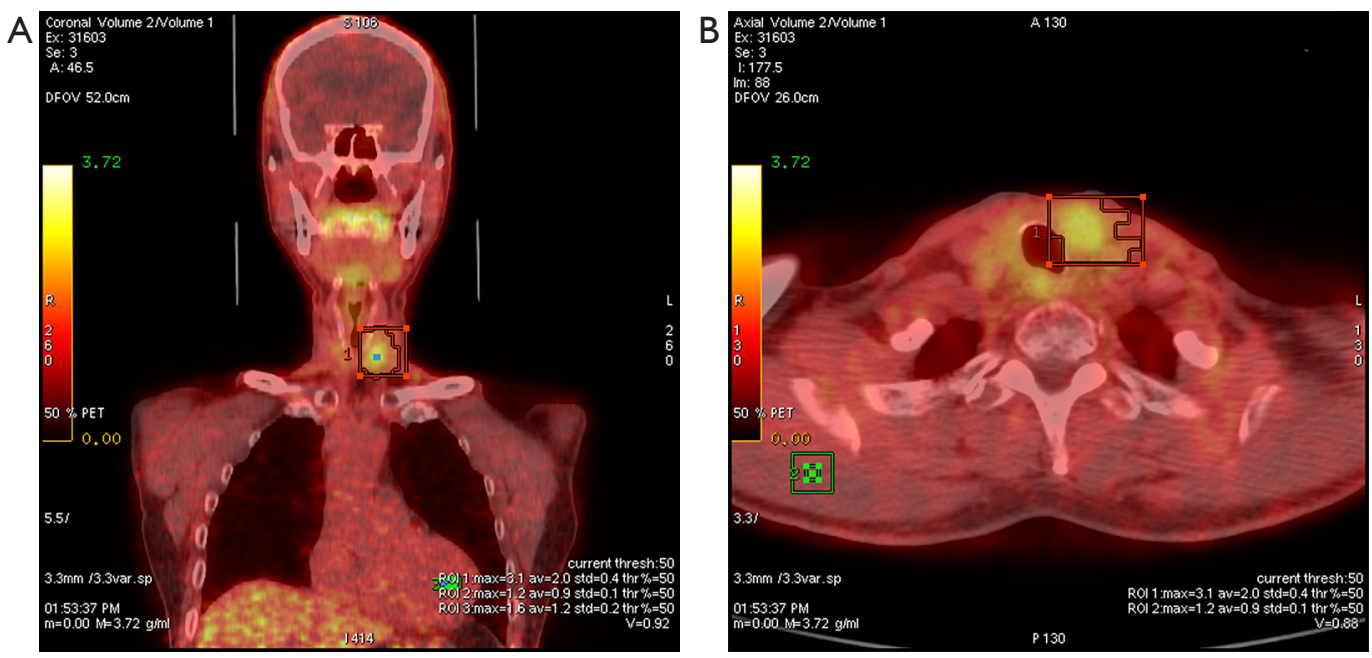

Figure 1 4-borono-s2- ${ }^{18}$ F-fluoro-phenylalanine positron emission tomography (FBPA-PET) in the coronal (A) and axial view (B), taken on August 10, 2018, two months before boron neutron capture therapy (BNCT). An increased FBPA uptake was noted in the left thyroid bed (highlighted square). The tumor-to-normal ratio (T/N) of the patient was 2.22. Images courtesy of the Nuclear Medicine Department, Taipei Veterans General Hospital.

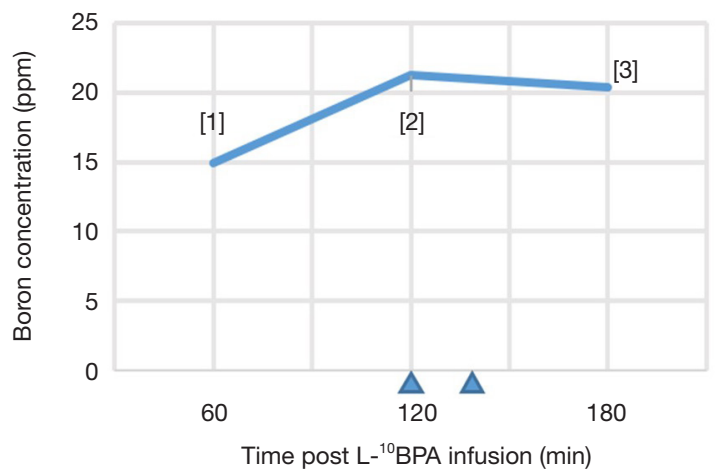

Figure 2 Blood boron concentrations at different time points during boron neutron capture therapy (BNCT), measured by inductively coupled plasma atomic emission spectrometer (ICP-AES). Courtesy of Professor Fong-In Chou's laboratory, National Tsing-Hua University. The patient's blood boron concentration was: [1] $14.92 \mathrm{ppm}$, at one hour after L-[4-10borophenylalanine] fructose (L-10BPA) infusion (180 mg/kg body weight per hour); [2] 21.27 ppm, at two hours after L-10BPA infusion (180 mg/kg body weight per hour); [3] 20.36 ppm, at three hours after L-10BPA infusion $(90 \mathrm{mg} / \mathrm{kg}$ body weight per hour). After two hours of L-10BPA infusion, the patient received epithermal neutron irradiation for about 17.05 minutes. The start and end points of irradiation interval was indicated by the two arrowheads.

8, 2018, BNCT was performed at the THOR, the only epithermal neutron source in Taiwan. A sono-guided fine needle biopsy was performed on the patient's left thyroid lesion to confirm the diagnosis of recurrent papillary thyroid carcinoma.

After initial position simulation, the patient received a total dose of $450 \mathrm{mg} / \mathrm{kg}$ of L-[4-10borophenylalanine] fructose (L-10BPA) via continuous intravenous infusion.
In order to maintain a constant blood boron concentration during irradiation, the infusion formulation was designed for full infusion during the first two hours and half rate during neutron irradiation (180 to 180 to $90 \mathrm{mg} / \mathrm{kg}$ body weight per hour) (Figure 2) (7). The blood boron concentration was $14.92 \mathrm{ppm}$ at 1 -hour post infusion and $21.27 \mathrm{ppm}$ at 2 hours post-infusion, just before epithermal neutron irradiation. After two hours of L-10BPA infusion, 

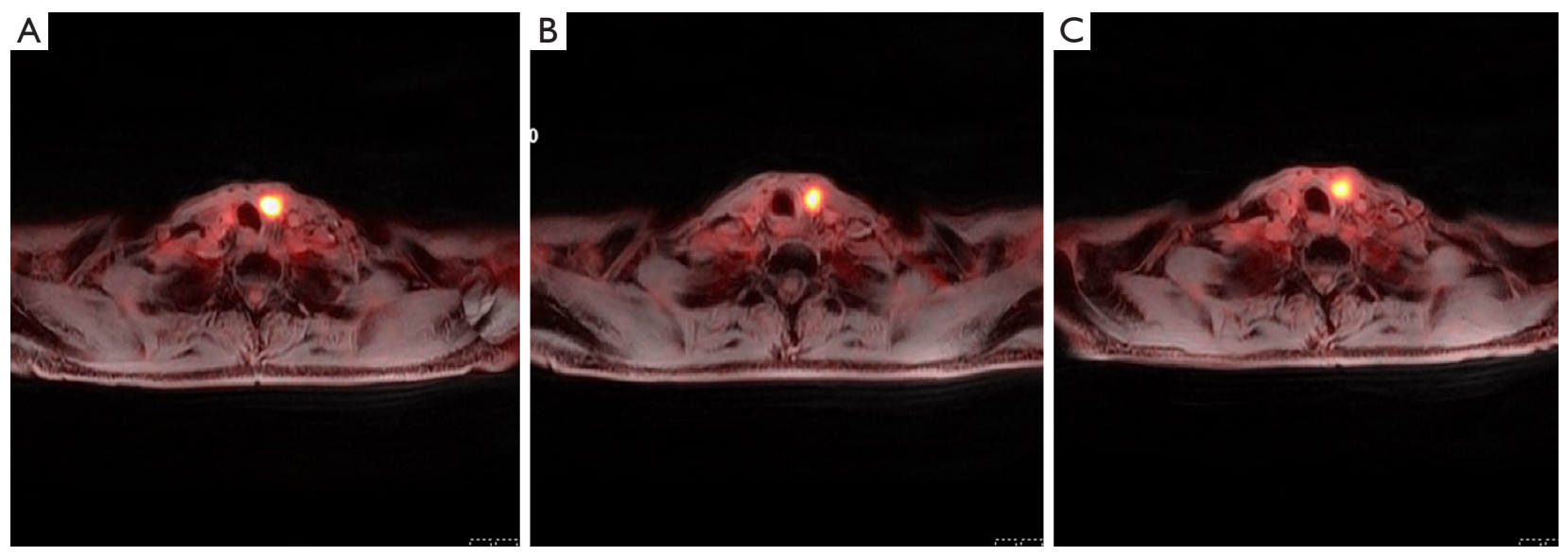

Figure 3 Comparisons of $2-{ }^{18}$ F-fluoro-2-deoxy-D-glucose positron emission tomography/magnetic resonance image (FDG-PET/MRI) before and after boron neutron capture therapy (BNCT). (A) The maximum standardized uptake value (SUVmax) was 17.8 on June 29 , 2018, four months before BNCT. (B) The SUVmax was 11.7 on November 8, 2018, one month after BNCT. (C) The SUVmax was 11.3 on March 27, 2019, five months after BNCT. Images courtesy of the Nuclear Medicine Department, Taipei Veterans General Hospital.

the patient then received epithermal neutron irradiation. With the reactor operating at 1.2 megawatts, the irradiation process took about 17.05 minutes with one portal. Gross tumor volume (GTV) received a mean dose of 22.36 gray equivalent (GyE), a minimal dose of $19.83 \mathrm{GyE}$ and a maximal dose of $23.60 \mathrm{GyE}$. The dosage in BNCT studies is calculated as the sum of three major contributors: the neutron dose, the boron dose, and the gamma dose. To account for the different biological effectiveness of these dose components, various weighting factors have been applied to generate a single, total weighted dose. They include relative biological effectiveness (RBE) for neutron, which was measured in animal models, and compound biological effectiveness (CBE) for boron drug, taking intraand intercellular heterogeneity of boron distribution into consideration. In this study, RBE was 3.2 for epithermal neutron, 1.0 for gamma; CBE for 10BPA was 4.9 for mucosa, 1.3 for normal tissue.

One month after BNCT, a PET/MRI scan on November 8, 2018 revealed evident regression of the recurrent tumor in the left thyroid region compared with pre-BNCT PET/ MRI, with the SUVmax decreasing from 17.8 to 11.7 (Figure 3). Thyroglobulin (Tg) also dropped significantly, from 55.6 to $15.1 \mathrm{ng} / \mathrm{mL}$ (Figure 4). The patient reported no significant side effects after BNCT. He has been followed-up at outpatient clinic every three months for one year till November 27, 2019. Thyroid sonography and MRI revealed no local recurrence nor distant metastasis. A brief clinical course of the patient was summarized in Figure 5.
Written informed consent was obtained from the patient for publication of this manuscript and any accompanying images. A copy of the written consent is available for review by the Editor-in-Chief of this journal.

\section{Discussion}

Among thyroid malignancies, papillary thyroid carcinoma is the most prevalent subtype, comprising $88.1 \%$ of cases for male thyroid cancer patients and $90.83 \%$ of those for females in Taiwan (2). An indolent clinical course is usually observed in this group. The mean age at the time of initial diagnosis is about $40-50$ years. In addition, it includes histopathologic variants defined by growth pattern (follicular, macrofollicular, cribriform-morular, solid), size (papillary microcarcinoma), or cellular characteristics (hobnail/ micropapillary, tall cell, oncocytic, columnar cell) (6).

The primary treatment for most types of thyroid tumors is surgical, either lobectomy or total thyroidectomy. Adjuvant RAI is usually administered 4-12 weeks after surgery, to destroy any remnant thyroid tissue or residual tumor. In spite of routine usage, adjuvant RAI is now selectively recommended if the largest primary tumor is $2-4 \mathrm{~cm}$, or if the patient has a high-risk histology (poorly differentiated, variants of tall cell, columnar cell and hobnail), lymphatic invasion, cervical node metastasis, macroscopic multifocality, postoperative unstimulated $\mathrm{Tg}$ $<5-10 \mathrm{ng} / \mathrm{mL}$, microscopic positive margins or positive findings on postoperative ultrasound. Furthermore, RAI 


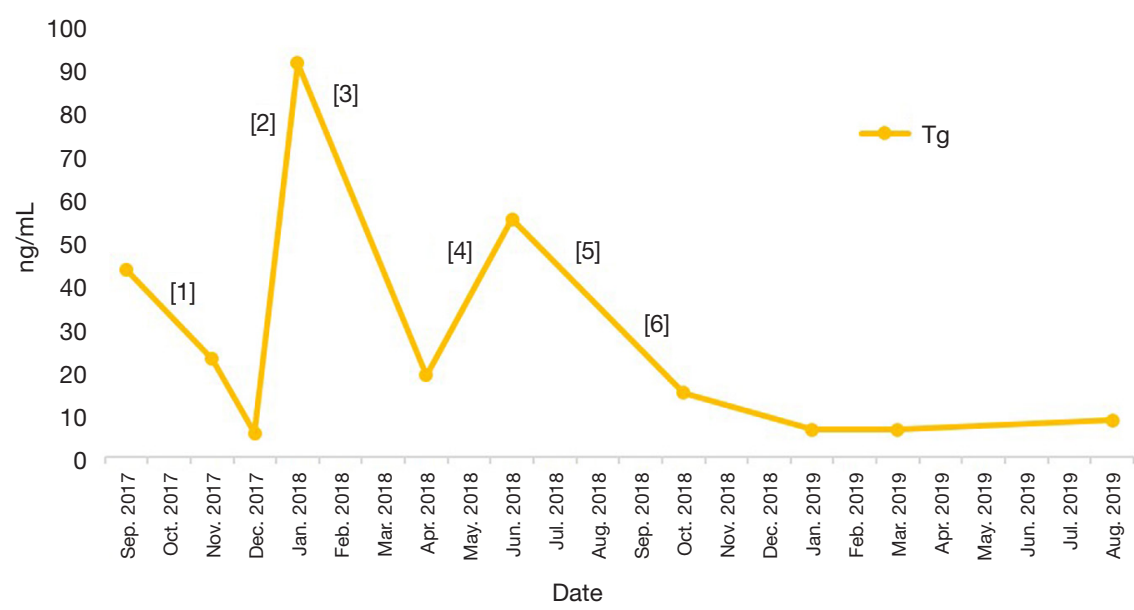

Figure 4 Serum thyroglobulin ( $\mathrm{Tg}$ ) levels during the clinical course, with the corresponding treatment time points. The patient received the following treatments at the indicated time points. [1] Tomotherapy with 55 gray (Gy)/29 fractions to the residual tumor and lymphadenopathies, and 45 Gy/25 fractions to the bilateral lower neck from September 21 to November 3, 2017. [2] Two doses of thyrogen $0.9 \mathrm{mg}(1 \mathrm{cc})$ were given intramuscularly, on January 24 and 25, 2018, in preparation for radioiodide thyroid cancer treatment. [3] Oral iodine-131 ablation therapy with dose of 30 millicuries ( $\mathrm{mCi}$ ) was given on January 26, 2018. [4] Two doses of thyrogen 0.9 mg (1 cc) were given intramuscularly, on June 26 and 27, 2018, in preparation for radioiodide thyroid cancer treatment. [5] Oral iodine-131 ablation therapy with a dose of $120 \mathrm{mCi}$ was given on June 28, 2018. [6] Boron Neutron Capture Therapy was performed on October 8, 2018.

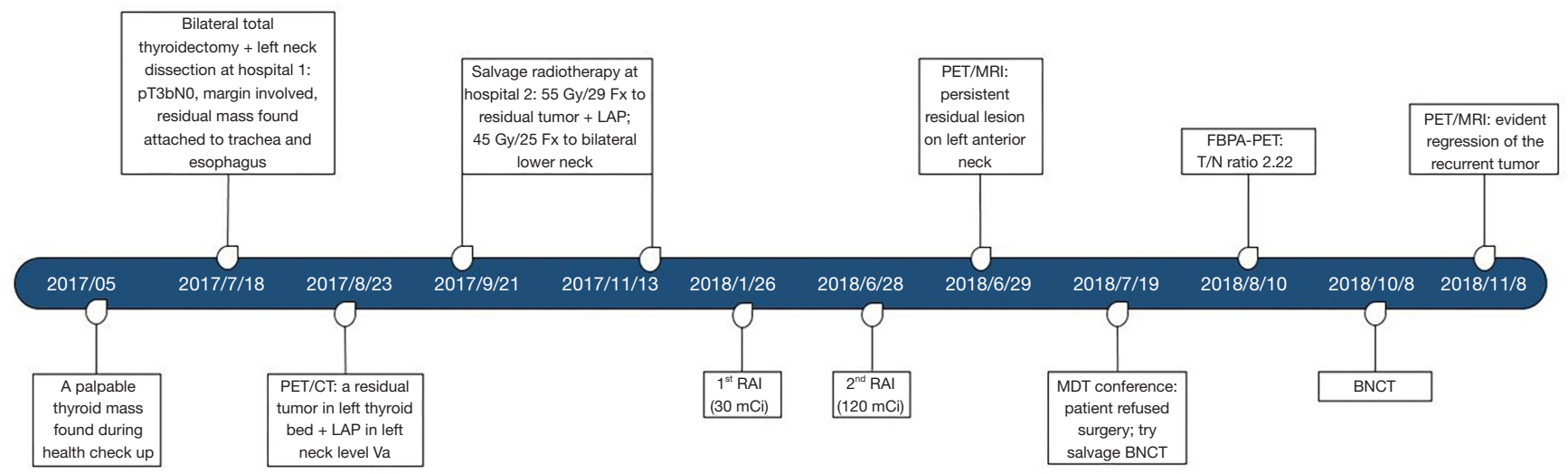

Figure 5 Summary of patient's clinical course in timeline. PET, positron emission tomography; CT, computed tomography; LAP, lymphadenopathy; Fx, fractions; RAI, radioactive iodine; $\mathrm{mCi}$, millicuries; MRI, magnetic resonance imaging; MDT, multidisciplinary team; BNCT, boron neutron capture therapy; FBPA, 4-borono-s2- ${ }^{18} \mathrm{~F}$-fluoro-phenylalanine; T/N ratio, tumor-to-normal ratio.

is typically suggested if there is any gross extrathyroidal extension; the primary tumor is $>4 \mathrm{~cm}$; the postoperative unstimulated $\mathrm{Tg}$ is $>5-10 \mathrm{ng} / \mathrm{mL}$; or there are bulky or numerous ( $>5$ ) positive lymph nodes (8).

As is the case for most DTCs, papillary thyroid carcinoma maintains the typical features of the thyroid follicular cell, which takes up I-131 via sodium-iodide transporter and causes cell death. Thus, it is usually curable with surgery and RAI. For long-term management and follow up, since it responds to thyroid-stimulating hormone (TSH), the suppression of TSH levels using supraphysiological doses of levothyroxine is recommended to decrease the rate of recurrence. In addition, the serum Tg level can also serve as a marker of recurrence or residual 
disease. Undetectable serum $\mathrm{Tg}$ levels can reliably identify disease-free patients, with a negative predictive value close to $100 \%(9)$.

In this case, our thyroid cancer patient had an intricate clinical course. Due to the lack of pre-operative imaging and the surgical note from the first local hospital, the definite initial cancer staging, extent of tumor invasion and completeness of resection cannot be ascertained. Consequently, the rationale for choosing external beam radiation therapy (EBRT) as a first-line adjuvant therapy rather than RAI was questionable. Our patient had a pT3b papillary thyroid carcinoma of the conventional histological type, with a microscopically positive resection margin and unknown status of the residual tumor. As reported in the literature (10), EBRT is generally advised as an additional treatment for unresectable, locally advanced or metastatic disease in primarily noniodine-avid thyroid tumors. Given the nature of conventional papillary thyroid carcinoma, our patient should have responded well to adjuvant RAI. Although prior retrospective studies have demonstrated survival benefits of adjuvant EBRT (11), its selective use is suggested only in certain DTC patients (those over age 60 years with initial extrathyroidal extension but without gross residual disease; or younger patients with T4b/extensive T4a disease and poor histologic features) $(12,13)$. An expert consensus from the 37th Annual Conference of European Society Radiation Oncology also stated the relevant indications for EBRT in DTC: pT4 tumors with a grossly positive margin (R2) and low/no RAI uptake; or surgically unresectable loco-regional recurrences with low/no RAI uptake (14). The role of EBRT in the postoperative setting of DTC remains controversial in the absence of prospective randomized clinical trials $(15,16)$. In contrast, the application of RAI in DTC patients has been well established (9).

Since the 2000s, combatting thyroid cancer with BNCT has been extensively studied. The first clinical trial of BNCT for thyroid cancer was reported by Hiratsuka et al. in 2006, who reported significant effects of BNCT on unresectable papillary thyroid carcinoma (17). A serial study by Dagrosa $e t a l$. also concentrated on treating UTC, with respect to the biochemical features of boron compounds and optimization of BNCT (4,5). Recently, Enomoto et al. revealed the overexpression in anaplastic thyroid cancer (ATC) of L-neutral amino acid transporter 1 protein, a key transporter of p-boronophenylalanine (BPA) into cancer cells, thus implying that BNCT may also be effective for ATC (18). So far, most investigations have been confined to UTC or poorly differentiated thyroid carcinoma (PDTC) due to the relatively aggressive characteristics of these cancers (19). However, recurrent DTC may share the same trait and be resistant to therapeutic doses of RAI, chemotherapy and radiation therapy. The actual mechanism has yet to be revealed. In our case, the patient received EBRT prior to RAI treatment. Hence, we postulated that the papillary carcinoma had lost its ability to uptake I-131, as is typical of thyroid follicular cells after receiving a tumoricidal dose of radiation. Indeed, Dagrosa et al. demonstrated in 2011 a similar level of BPA uptake between DTC cells and UTC cells, both in vitro and in cells implanted in nude mice (20). After our patient received BNCT on October 8, 2018, the serum Tg value had decreased from $55.6 \mathrm{ng} / \mathrm{mL}$ (June 28,2018 ) to $15.1 \mathrm{ng} / \mathrm{mL}$ (October 24, 2018) and continued to decline. On the other hand, the $\mathrm{T} / \mathrm{N}$ ratio for our case showed 2.22, below the expected 2.5-fold. However, because thyroid tumors are usually located at the surface of the neck, patients who presents with a low $\mathrm{T} / \mathrm{N}$ ratio can also be treated well if high neutron flux is provided. PET/MRI on March 25, 2019 showed stable disease compared to the previous image taken on November 8, 2018. Follow up at our clinic showed no relapse according to thyroid function test and thyroid gland ultrasound until August 2019. The patient continued to receive levothyroxine supplements and no side effects were observed by clinicians. This case study is subject to some limitations. First, we had limited access to the patient's full medical records in two other hospitals. It hindered us from retrieving decisive details nor bridging the gaps between treatment modalities. Second, papillary thyroid tumor has been known for a rather curable disease and succumbs to therapy that were extensively studied. In contrast, there was a lack of previous research on treating recurrent or refractory papillary thyroid tumor by other management. Thus, further investigation needs to be conducted in order to explicitly evaluate the efficiency of salvage BNCT.

In conclusion, for recurrent or refractory thyroid cancer, BNCT can be used as a salvage treatment. Clinical efficacy can be achieved with no effect on the patient's quality of life. Based on the result of this case, BNCT is effective for the treatment of recurrent thyroid papillary carcinoma.

\section{Acknowledgments}

The research group would like to express the highest gratitude to the following researchers who provided assistance for the study: Professor Shiang-Huei Jiang, 
Yen-Wen Liu Hsieh (providing THOR plan as treatment planning system) and Dr. Hong-Ming Liu from Institute of Nuclear Engineering and Science, Tsing-Hua University; Taiwan Biotech Company for manufacturing of L-BPA; Chi-Wei Chang, Nuclear Pharmacist of Department of Nuclear Medicine, Taipei Veterans General Hospital; Study Nurse: Pei-Yu Lai, and research assistant: Meng-Hsuan Lin; Research Center for Boron Neutron Capture Therapy at Tamkang University.

Funding: None.

\section{Footnote}

Reporting Checklist: The authors have completed the CARE reporting checklist. Available at http://dx.doi.org/10.21037/ tro-20-10

Conflicts of Interest: All authors have completed the ICMJE uniform disclosure form (available at http://dx.doi. org/10.21037/tro-20-10). YWC serves as an unpaid editorial board member of Therapeutic Radiology and Oncology from Apr 2020 to Mar 2022. The other authors have no conflicts of interest to declare.

Ethical Statement: The authors are accountable for all aspects of the work in ensuring that questions related to the accuracy or integrity of any part of the work are appropriately investigated and resolved. All procedures performed in studies involving human participants were in accordance with the ethical standards of the institutional and/or national research committee(s) and with the Helsinki Declaration (as revised in 2013). Written informed consent was obtained from the patient for publication of this manuscript and any accompanying images.

Open Access Statement: This is an Open Access article distributed in accordance with the Creative Commons Attribution-NonCommercial-NoDerivs 4.0 International License (CC BY-NC-ND 4.0), which permits the noncommercial replication and distribution of the article with the strict proviso that no changes or edits are made and the original work is properly cited (including links to both the formal publication through the relevant DOI and the license). See: https://creativecommons.org/licenses/by-nc-nd/4.0/.

\section{References}

1. Kim J, Gosnell JE, Roman SA. Geographic influences in the global rise of thyroid cancer. Nat Rev Endocrinol 2020;16:17-29.

2. 2016 Annual Cancer Registration Report in Taiwan, Health Promotion Administration Ministry of Health and Welfare, Taiwan (December 27th 2018).

3. Lamartina L, Grani G, Durante C, et al. Recent advances in managing differentiated thyroid cancer. F1000Res 2018;7:86.

4. Dagrosa MA, Thomasz L, Longhino J, et al. Optimization of boron neutron capture therapy for the treatment of undifferentiated thyroid cancer. Int J Radiat Oncol Biol Phys 2007;69:1059-66.

5. Dagrosa M, Crivello M, Thorp S, et al. Radiobiological studies in a human cell line of undifferentiated thyroid cancer. Proceedings of 13 ICNCT, Florence, 2008:337-40.

6. Tallini G, Giordano TJ, Gland T. In: Rosai J, Ackerman LV. editors. Rosai and Ackerman's surgical pathology. Mosby, Edinburgh, 2011:278-334.

7. Miyatake S, Kawabata S, Yokoyama K, et al. Survival benefit of Boron neutron capture therapy for recurrent malignant gliomas. J Neurooncol 2009;91:199-206.

8. National Comprehensive Cancer Network. (2019). Thyroid cancer (version 2.2019). Available online: https:// www.nccn.org/professionals/physician_gls/pdf/thyroid.pdf

9. Haugen BR, Alexander EK, Bible KC, et al. 2015 American Thyroid Association Management Guidelines for Adult Patients with Thyroid Nodules and Differentiated Thyroid Cancer: The American Thyroid Association Guidelines Task Force on Thyroid Nodules and Differentiated Thyroid Cancer. Thyroid 2016;26:1-133.

10. Terezakis SA, Lee KS, Ghossein RA, et al. Role of external beam radiotherapy in patients with advanced or recurrent nonanaplastic thyroid cancer: Memorial Sloan-kettering Cancer Center experience. Int J Radiat Oncol Biol Phys 2009;73:795-801.

11. Fussey JM, Crunkhorn R, Tedla M, et al. External beam radiotherapy in differentiated thyroid carcinoma: A systematic review. Head Neck 2016;38 Suppl 1:E2297-E2305.

12. Yang Z, Flores J, Katz S, et al. Comparison of Survival Outcomes Following Postsurgical Radioactive Iodine Versus External Beam Radiation in Stage IV Differentiated Thyroid Carcinoma. Thyroid 2017;27:944-52.

13. Tam S, Amit M, Boonsripitayanon M, et al. Adjuvant External Beam Radiotherapy in Locally Advanced Differentiated Thyroid Cancer. JAMA Otolaryngol Head Neck Surg 2017;143:1244-51.

14. Blais E, Lagarde P, Henriques de Figueiredo B, et al. 
Indications for external beam radiotherapy in differentiated thyroid carcinoma: an expert consensus. Radiother Oncol 2018;127:S653-S654.

15. Kiess AP, Agrawal N, Brierley JD, et al. External-beam radiotherapy for differentiated thyroid cancer locoregional control: A statement of the American Head and Neck Society. Head Neck 2016;38:493-8.

16. Kim YS, Choi JH, Kim KS, et al. The role of adjuvant external beam radiation therapy for papillary thyroid carcinoma invading the trachea. Radiat Oncol J 2017;35:112-20.

17. Hiratsuka J, Morita N, Aihara T, et al. First clinical trial of boron neutron capture therapy for thyroid cancer. In: Nakagawa Y, Kobayashi T, Fukuda H, et al. editors.

doi: $10.21037 /$ tro-20-10

Cite this article as: Pan YY, Yao SF, Lin KH, Chou FI, Lee JC, Tai SK, Huang WS, Lan KL, Chao Y, Chen YW. Boron neutron capture therapy as salvage treatment for recurrent papillary thyroid carcinoma-a case report. Ther Radiol Oncol 2020;4:21.
Advances in neutron capture therapy. Proceedings of 12 th international congress on neutron capture therapy, 2006:638.

18. Enomoto K, Sato F, Tamagawa S, et al. A novel therapeutic approach for anaplastic thyroid cancer through inhibition of LAT1. Sci Rep 2019;9:14616.

19. Rodriguez C, Carpano M, Curotto P, et al. In vitro studies of DNA damage and repair mechanisms induced by BNCT in a poorly differentiated thyroid carcinoma cell line. Radiat Environ Biophys 2018;57:143-52.

20. Dagrosa A, Carpano M, Perona M, et al. Studies for the application of boron neutron capture therapy to the treatment of differentiated thyroid cancer. Appl Radiat Isot 2011;69:1752-5. 